\title{
Erfassung von Brachland in der zukünftigen Arealstatistik
}

\section{Erneuerung der Arealstatistik}

Anfangs 1984 beginnt das Bundesamt für Statistik mit der Erneuerung der mit verschiedenen Mängeln behafteten, aus dem Jahre 1972 stammenden Arealstatistik. Es ist vorgesehen, in den Jahren 1984-86 eine vollständige Neuerhebung der Landnutzung durchzuführen. Ab 1987 werden die Daten im Turnus von sechs Jahren nachgeführt. Die Erhebungen erfolgen mittels eines speziell für eine großräumige Erhebung entwickelten Verfahrens der stichprobenweisen Auswertung von Luftaufnahmen (1). Ein Stichprobennetz von 100 Metern Maschenweite wird dabei rechnerisch der Geometrie der Luftbilder angepaßt. Mit einer computergesteuerten Zeichenanlage wird der projektiv verzerrte Stichprobenraster auf eine transparente Folie gezeichnet. Diese wird den Luftbildern überlagert, und für jeden Stichprobenpunkt wird aufgrund einer detaillierten, rund 40 Kategorien umfassenden Landnutzungsklassifikation (vgl. Abb.1) die jeweilige Bodennutzung bestimmt. Die Resultate dieser Erhebungen werden in der Flächendatei (Hektarraster) des Informationsrasters beim Bundesamt für Statistik gespeichert, ausgewertet und verwaltet.

Da sich diese Erhebungen für die Arealstatistik über das ganze Territorium der Schweiz erstrecken, stellt sich zwangsläufig die Frage, wie Brachflächen dabei berücksichtigt werden sollen.

Im folgenden Abschnitt wird zuerst auf die Möglichkeiten der Erfassung von Brachland mit Hilfe von Luftbildern eingegangen, anschließend wird gezeigt, wie Brachflächen in der neuen Arealstatistik berücksichtigt werden, und zum Schluß werden einige Anregungen im Hinblick auf zukünftige Brachlanderhebungen gemacht.

\section{Möglichkeiten zur Erfassung von Brachland mit Hilfe von Luftaufnahmen}

Im Rahmen eines gemeinsamen Projektes der Eidg. Anstalt für das forstliche Versuchswesen sowie des ORL-Institutes ergab sich 1977 die Möglichkeit, ein Testgebiet mehrmals befliegen zu lassen, um offene Fragen im Zusammenhang mit der Erfassung von Brachflächen auf Luftaufnahmen zu bearbeiten.
Folgende Fragen standen dabei im Vordergrund:

- lassen sich verschiedene Brachlandphasen (Gras-, Stauden- und Strauchphasen) im Luftbild erkennen?

- welche Jahreszeit eignet sich dazu am besten?

- welches ist das geeignetste Filmmaterial?

- welches ist der günstigste Bildmaßstab?

Als Testgebiet wurde die Gemeinde Merishausen (Kt. Schaffhausen) gewählt. $\mathrm{Zu}$ drei unterschiedlichen Zeitpunkten (26.4., 7.7., 4.10.77) wurden Schwarzweiß-, Farb- und Infrarot-Farbaufnahmen in den Maßstäben $1: 10000$ und $1: 25000$ hergestellt (2). Diese Luftbilder wurden bezüglich ihres Informationsgehaltes für die Erhebung von Brachflächen untersucht. Die Ergebnisse können wie folgt zusammengefaßt werden (3):

- Im Frühling lieferten alle drei Filmtypen günstige Voraussetzungen für eine Ausscheidung von frühen Brachlandphasen (holzfreie Sukzessionsphasen: Gras- und Staudenphasen). Im Vorjahr nicht genutzte Flächen, die über einen dichten Streufilz verfügen, treten auf den Luftbildern deutlich in Erscheinung. Hingegen hat sich gezeigt, da $\beta$ auch im Maßstab 1:10000 keine weitere Unterteilung dieser holzfreien Sukzessionsphasen in Gras- und Staudenphasen möglich ist. Auch junge Strauchphasen (kleiner als ca. $1 \mathrm{~m}$ ) konnten nicht mit Sicherheit erkannt werden. Bezüglich der Beurteilung der Verbuschung (fortgeschrittenere Brachlandphasen) eignen sich Frühlingsaufnahmen nicht, wenn sie vor der Belaubung aufgenommen wurden.

- Auf den im Sommer aufgenommenen Luftbildern konnten frühe Brachlandphasen nicht ausgeschieden werden; hingegen war die Beurteilung der Verbuschung besser möglich.

- Im Herbst bildeten die Infrarot-Farbaufnahmen die besten Voraussetzungen unter den drei Filmtypen für die Ausscheidung von holzfreien Sukzessionsphasen. Bezüglich der weiteren Unterteilung der frühen Brachlandphasen gilt das gleiche wie bei den Frühlingsaufnahmen.

Dr. Heinz Trachsler, ORL-Institut ETH Zürich, 8093 Zürich 
Diese Angaben beziehen sich auf das Testgebiet Merishausen. Es müßte zuerst abgeklärt werden, ob sie auch auf andere Gebiete übertragbar sind. Im Alpenraum ergeben sich auf jeden Fall zeitliche Verschiebungen bezüglich der günstigsten Aufnahmeperioden infolge der längeren Schneebedeckung.

Bei diesen Ergebnissen ist weiter zu beachten, daß die Luftbilder als Momentaufnahmen nur erlauben, Flächen auszuscheiden, die von ihrem Aspekt her den Charakter von Brachland haben. Aufgrund dieser Bilder kann jedoch nicht mit Bestimmtheit gesagt werden, ob diese Flächen langfristig auch tatsächlich nicht genutzt werden. Dazu sind ergänzende Untersuchungen in Form von Befragungen oder Feldbegehungen notwendig.

Aus den Untersuchungen in Merishausen wird deutlich, daß eine Erhebung der aus planerischer Sicht interessanten Flächen mit Gras-, Stauden- und frühen Strauchphasen, bei denen aus forstgesetzlicher Sicht noch keine Nutzungseinschränkungen bestehen, nur während relativ kurzer Zeit möglich ist. Die Landnutzungserhebungen der Arealstatistik müssen aus Kostengründen mit den Schwarzweiß-Luftbildern 1:25000 des Bundesamtes für Landestopographie durchgeführt werden. Diese Bilder sind jedoch bezüglich des Aufnahmezeitpunktes und z.T. auch hinsichtlich des Filmmaterials (Herbst) nicht oder nur beschränkt geeignet für eine landesweite Erfassung dieser frühen Brachlandphasen. Es können damit lediglich Flächen mit fortgeschrittenen Brachlandphasen, die eindeutig anhand von Sträuchern und Gebüschen identifiziert werden können, erhoben werden. Im Hinblick auf andere Nutzungsmöglichkeiten handelt es sich dabei mehrheitlich um irreversible Brachflächen.

\section{Berücksichtigung von Brachflächen in der neuen Arealstatistik}

Abb. 1 zeigt den voraussichtlich für die Arealstatistik zur Anwendung kommenden Kategorienkatalog (4). Bei dessen Erarbeitung wurden sowohl die Wünsche der potentiellen Benützer der Statistik als auch Randbedingungen aus der Sicht der Erhebungsmethode berücksichtigt. Bei einer Umfrage bezüglich der aus Benützersicht wünschbaren Kategorien in einer neuen Arealstatistik wurde von $20 \%$ der Befragten der Wunsch geäussert, Brachflächen gesondert zu erheben. Im Rahmen der insgesamt etwa 45 Stellungnahmen aus dem Vernehmlassungsverfahren zur vorgeschlagenen Erhebungsmethode und zum neuen Kategorienkatalog wurde lediglich einmal der Wunsch geäußert, eine spezielle Kategorie Brachland einzuführen.

Einschränkungen aus der Sicht der Erhebungsmethode wurden bereits in Abschnitt 2 behandelt, demnach muß auf eine gesonderte Erfassung von Gras-, Stauden- und jungen Strauchphasen verzichtet werden. Diese Flächen sind entweder im Weideland oder beim Wiesland enthalten.

Abb. 1 zeigt, daß eine eigentliche Kategorie «Brachland» in der Statistik fehlt. Wie bereits erwähnt, erlaubt es das Luftbild nur, das Erscheinungsbild einer Fläche zu beurteilen, also z. B. ob sie verbuscht ist. Aufgrund des Luftbildes kann jedoch nicht mit Bestimmtheit gesagt werden, ob diese Fläche durch Brachlegung entstanden ist. Aus diesem Grund wurde bewußt auf die Verwendung des Begriffs Brachland verzichtet. Trotzdem enthält die Statistik gewisse Informationen über Brachflächen. Flächen, die den Aspekt von fortgeschrittenen Brachlandphasen aufweisen (Strauchphasen, Baumphasen), sind in den folgenden Kategorien (vgl. auch Abb. 1) enthalten:

- verbuschte Alpweiden

- Gebüsch (Oberhöhe $<3 \mathrm{~m}$ )

- Gebüschwald (Definition gemäß Schweizerischem Landesforstinventar: Oberhöhe $<3 \mathrm{~m}$, mindestens $\mathrm{zu} 2 / 3$ mit Sträuchern bedeckt).

Aufgrund des vorliegenden Kategorienkataloges ist nach Abschluß der erstmaligen Erhebung der Arealstatistik somit nur das Ausmaß der Flächen bekannt, die den Aspekt von fortgeschrittenen Brachlandphasen aufweisen. Wieweit diese Flächen tatsächlich durch Brachlegung entstanden sind, müßte durch zusätzliche Untersuchungen, z.B. Vergleichen mit älteren Luftaufnahmen, abgeklärt werden. Bei der zukünftigen Nachführung der Arealstatistik wird sich hingegen die Aussagekraft bezüglich der Brachflächen erhöhen. Dabei wird sich nämlich zeigen, wie und wo sich die drei "Brachland-Kategorien» verbuschte Alpweiden, Gebüsch und Gebüschwald verändert haben. Wichtig ist dabei auch, daß ersichtlich wird, aufgrund von welchen anderen Nutzungskategorien allfällige Zunahmen bei diesen Kategorien entstanden sind. Bei der zukünftigen Nachführung der Arealstatistik können somit allfällige Brachlegungsprozesse im Bereich der fortgeschrittenen Brachlandphasen lokalisiert und flächenhaft erfaßt werden.

Da die Bodennutzungsdaten der Arealstatistik in Zukunft in der Flächendatei des Informationsrasters gespeichert sind (vgl. Abschn. 1), haben sie nicht mehr nur dokumentarischen Charakter. Dem Benützer eröffnen sich vielfältige Auswerte- und Abfragemöglichkeiten, wie z.B. Verknüpfung mit anderen Daten, Auswertung nach bestimmten Kriterien, graphische Darstellungen usw. 


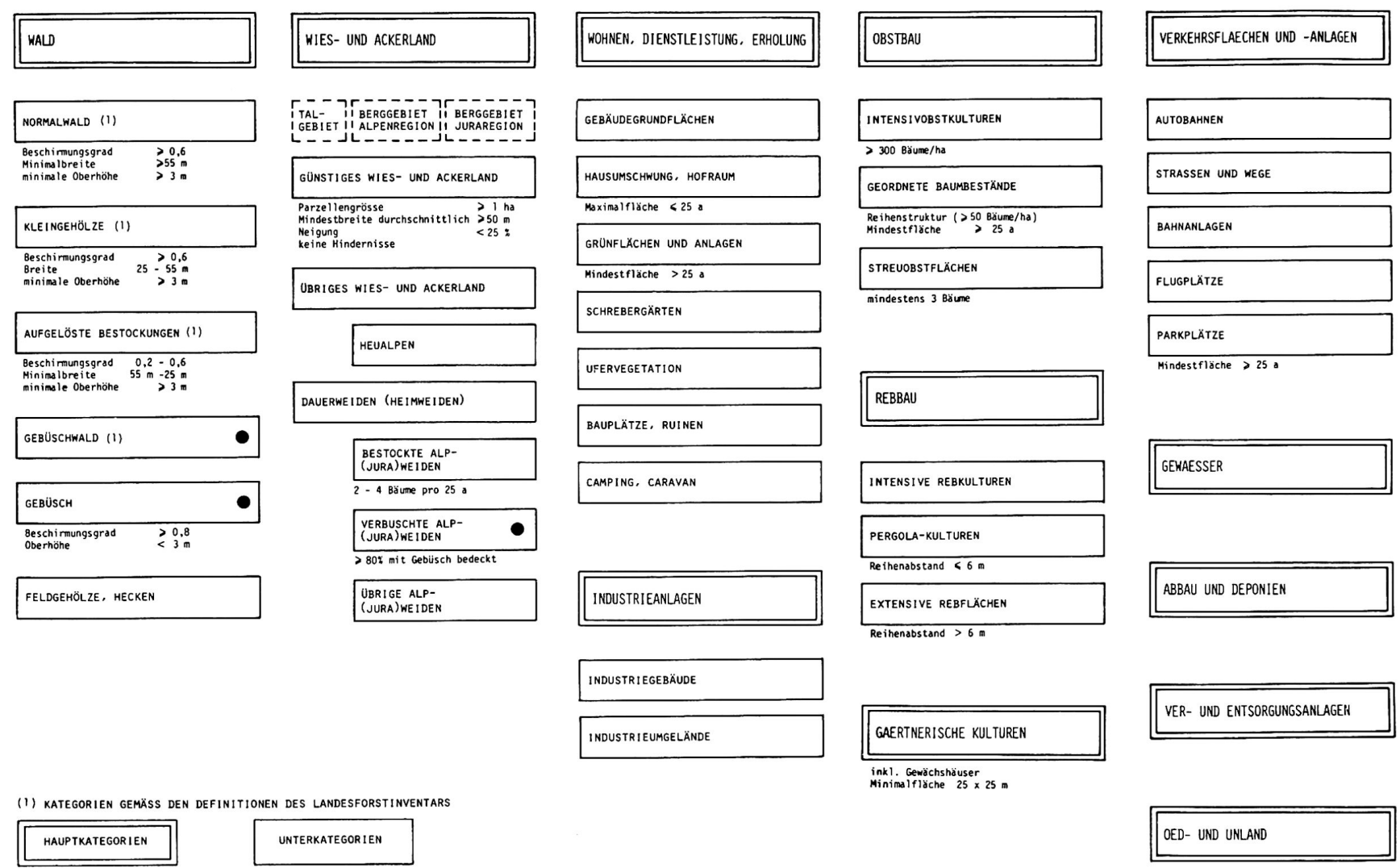

Abb. 1 Zusammenstellung der für die Erneuerung der Arealstatistik vorgesehenen Nutzungskategorien (vgl. auch Anmerkung 4). Flächen mit fortgeschrittenen Brachlandphasen sind in den mit markierten Kategorien enthalten. 


\section{Wünsche seitens der Arealstatistik an zukünftige Brachlanduntersuchungen}

Die bisherigen Ausführungen haben gezeigt, daß in absehbarer Zeit mit der Arealstatistik erstmals ein Instrument zur Verfügung stehen wird, das bis zu einem gewissen Grad Aussagen über Landschaftsveränderungen infolge Brachlegung ermöglichen wird. Allerdings wird die neue Arealstatistik im Bereich des Brachlandes noch in zweifacher Hinsicht Lücken aufweisen:

a) frühe Brachlandphasen werden nicht berücksichtigt.

b) aufgrund der Ersterhebung ist nicht mit Sicherheit zu sagen, wieweit die in Abschn. 3 erwähnten «Brachlandkategorien» tatsächlich durch Brachlegung entstanden sind.

\footnotetext{
Anmerkungen

(1) Eine ausführliche Beschreibung des Verfahrens der stichprobenweisen Auswertung von Luftaufnahmen befindet sich in:

TRACHSLER, H., KÖLBL, O., MEYER, B., MAHRER, F.: Stichprobenweise Auswertung von Luftaufnahmen für die Erneuerung der Eidgenössischen Arealstatistik - Bericht über einen Versuch in verschiedenen Testgebieten der Schweiz. Arbeitsdokumente für die schweizerische Statistik, Heft 5, Bundesamt für Raumplanung, Bern 1981

sowie:

MEYER, B., KÖLBL, O., TRACHSLER, H.: Neue Arealstatistik für die Schweiz. Vermessung, Photogrammetrie, Kulturtechnik, 10, 1982.
}

Aus der Sicht der Arealstatistik wäre es daher wünschenswert, wenn zukünftige lokale oder regionale Erhebungen, die auch frühe Brachlandphasen berücksichtigen, methodisch so konzipiert würden, daß sie in die Arealstatistik integriert und später auch nachgeführt werden könnten. Auf diese Weise könnte die Aussagekraft der Arealstatistik bezüglich Brachland nach und nach erhöht werden.

Da die unter b) erwähnten Lücken nur durch umfangreiche Zusatzerhebungen geschlossen werden könnten, wäre es wünschenswert, wenn zumindest anhand einzelner ausgewählter Testgebiete modellhaft aufgezeigt werden könnte, wieweit die Kategorien verbuschte Alpweiden, Gebüsch, Gebüschwald und allenfalls Wald tatsächlich durch Brachlegung entstanden sind.
(2) Die Luftaufnahmen wurden uns in verdankenswerter Weise vom Flugdienst der Eidg. Vermessungsdirektion zur Verfügung gestellt.

(3) Eine ausführliche Beschreibung findet sich in:

SCHERRER, H.U., TRACHSLER, H., GRESCH, P.: Vorschläge zur Kartierung und raumplanerischen Behandlung von Brachflächen. Eidg. Anstalt für das forstliche Versuchswesen, Berichte Nr. 207, Birmensdorf 1980.

(4) Dieser Kategorienkatalog wurde im Rahmen eines umfangreichen Versuches erprobt. Aufgrund einer anschließend durchgeführten Vernehmlassung unter den potentiellen Benützern wird er noch geringfügige Änderungen erfahren. 